\title{
CÓMO APRESAR LAS COMPETENCIAS DEL BEBÉ MEDIANTE UNA APLI- CACIÓN DE LA METODOLOGÍA OBSERVACIONAL
}

\author{
M. Teresa Anguera Argilaga \\ Facultad de Psicología, Universidad de Barcelona
}

RESUMEN. De entre las metodologías existentes el artículo presenta la metodología observacional (en contextos naturales o habituales) que es la que más posibilidades de aplicación ofrece al investigador para apresar las competencias de los bebés. Como procedimiento científico, permite poner de manifiesto la ocurrencia de conductas perceptibles, posibilita su registro, cuantificación y análisis de las relaciones existentes entre ellas. Entre sus ventajas, se destaca no sólo su ajuste a la particularidad del comportamiento del bebé, sino también la de permitir apresar y objetivar sus conductas en el continuum natural de su ocurrencia.

Se explican los requisitos necesarios para: 1) preparar la observación, 2) elaborar instrumentos (sistema de categorías, formatos de campo, sistema mixto y "rating scales"), 3) decidir la modalidad de registro según su nivel de sistematización, 4) garantizar el control de la calidad de datos y, 5) los diseños observacionales posibles (diacrónico, sincrónico y mixto o "lag-log"), destacando entre ellos como los más completos y potentes a los diseños "lag-log".

ABSTRACT. Among the actual methodologies, the observational one (in natural contexts) is presented as the most usefull because it allows us: to show and capture detectable behaviours, to quantify and to analyse the relationships among them. Specifically, it stands up, not only its fitting to the baby's characteristics, butr the possibilty to study them in their occurrence.

The article explains the necessary requisites for: 1) preparing the observation; 2) elaborating the control tools; 3) electing the registrying ways depending on the systematization levels; 4) warranting data's quality, and, 5) the possible observational designs standing out among them the "lag-log" designs as the most complete ones.

"Pendant que Gesell soumettait le bebé à diverses épreuves et que les caméras tournaient, j'essayais d'observer. Je prenais des notes. Puis, les films une fois développés, je comparais mes observations aux faits enregistrés par le caméra. Et je découvrais, à la fois déçu et ravi, la pauvreté, les erreurs, l'imbécilité de mon regard et l'intelligence de la caméra. J'apprenais à observer, j'apprenais aussi que l'observation est trompeuse à qui n'est pas foncièrement honnête, j'apprenais aussi que l'observation a des limites très étroites quand elle n'est pas guidée par des connaissances préalables et aidée 
par des techniques rigoreuses. J'apprenais la rigueur non pas des schémas rigides mais la rigueur des nuances. Je découvrais sans aucun discours l'infinie diversité des gestes de l'enfant, et qu'un individu, fût-il un nouveau-né, fût-il un jumeau, n'est jamais identique à un autre, même dans ses réactions les plus simples et les plus archaïques". [Zazzó (1964), en Mucchielli (1974, p. 6)]

\section{Adecuación metodológica}

Indudablemente es complejo apresar las competencias del bebé. Su sonrisa, su Ilanto, su mirada desvanecida, sus movimientos incoordinados,... no son fáciles de caracterizar, menos de registrar, y para muchos investigadores tampoco lo son de analizar con objetividad y rigor. Podemos añadir que menos aún si nos interesa estudiar la interacción entre ellos. Y la dificultad todavía aumenta en la medida en que, como es lógico, nos interese contemplar al bebé en los diversos contextos naturales / habituales en los cuales se halla inmerso (Sastre y Pastor, 1999).

Entre las diferentes opciones metodológicas existentes, indudablemente es la metodología observacional la que ofrece más posibilidades de aplicación, maximizándose sus ventajas y adquiriendo escasa relevancia los inconvenientes que le son inherentes.

La metodología observacional, que se desarrolla en contextos naturales o habituales, consiste en un procedimiento científico que pone de manifiesto la ocurrencia de conductas perceptibles, posibilitando su registro organizado y su cuantificación mediante un instrumento adecuado y parámetros convenientes, y permitiendo analizar las relaciones de diverso orden (secuencialidad, asociación, covariación, etc.) existentes entre ellas. Estas conductas, por la espontaneidad o habitualidad con que ocurren, pondrán de manifiesto todos aquellos elementos que se requieren destacar para proceder a su objetivación adecuada.

Son muchas las situaciones que giran en torno al bebé en las cuales la metodología observacional es la más adecuada o la única posible, como valoración de programas de intervención precoz (problemas de no aparición del habla en la edad adecuada, postura incorrecta al andar, rictus repetitivos, etc.), interacciones diversas (entre iguales, entre niños y adultos, entre sordos y oyentes, etc.), competencia social en edades diversas (niños solitarios, etc.), repertorio conductual del bebé, análisis del movimiento en diversas actividades, ocupación de un espacio determinado, pautas de socialización, etc.

La observación de las conductas del bebé en un contexto determinado requiere ajustarse a unos pocos requisitos para poderse materializar, y los diferenciaremos en sus dos vertientes sustantiva y metodológica:

1. La primera decisión a adoptar, la de carácter sustantivo, consistirá en la delimitación temática del comportamiento perceptible del bebé que nos interese analizar, y al cual afectarán tres únicas restricciones:

a) Su carácter perceptible, sea de forma total o parcial. Mucho se ha discutido sobre la perceptividad, y se han fijado posicionamientos en función de escuelas psicológicas en las que se establece un anclaje o referente al efecto. Nuestra posición es clara en el sentido de que abogamos por la consideración de con- 
ductas manifiestas del bebé -que implican grado total de perceptividad-, y que ofrecen una mayor garantía en su acotación.

b) El hecho de que forme parte de la vida cotidiana y del entorno natural del bebé. No tendría sentido el estudio de aspectos incidentales o anecdóticos, sino que interesa esencialmente el estudio de conductas espontáneas.

c) Relación interactiva con el entorno. Cualquier conducta requiere de un referente que tiene en cuenta el entorno en alguna de sus múltiples acepciones, y que aquí entendemos como el conjunto molar compuesto por los lugares definidos en el espacio en que ocurren diferentes actividades del bebé. Determinados entornos ofrecen unas especiales condiciones idóneas para la detección de necesidades: familia, escuela maternal, centro de acogida, etc.

2. Desde el punto de vista procedimental o metodológico, nos debemos plantear igualmente unos determinados requisitos perfectamente exigibles:

a) Preferencia por el carácter idiográfico del estudio. De esta forma se desarrollan mejor las posibilidades de la metodología observacional cuando se observa un bebé, que es la posición clásica, pero también cuando se hace uso de dos nuevas acepciones del concepto idiográfico: Por una parte, un pequeño grupo de individuos que actúan como una unidad (por ejemplo, interacción diádica madre-hijo, etc.), y, por otra, cuando solamente se observan conductas pertenecientes a un solo nivel de respuesta, tanto si corresponden a un solo sujeto (conducta de desplazamiento del bebé en un espacio), como al mencionado grupo de individuos que actúan como una unidad (conducta postural en grupos de cuatro niños mientras realizan una actividad de rítmica infantil).

b) Posibilidad de un cierto seguimiento temporal, que va más allá de una mera ocurrencia casual o esporádica de determinadas conductas. Es obvio adoptar un planteamiento dinámico en el estudio del comportamiento infantil, que hará conveniente fijar unos límites temporales que acoten el flujo interactivo, así como estudiar diacrónicamente determinados episodios de conducta.

c) Ausencia de instrumento estándar. El carácter espontáneo de las conductas que se estudian, así como el carácter habitual del contexto, imposibilitan la existencia previa de unos intrumentos encorsetados y estándar, que obligarían a limitar el carácter natural de las conductas producidas. Por dicho motivo, deberán construirse ad hoc en cada caso, con la excepción de las rating scales.

\section{Preparación de la observación: Observación exploratoria, requisitos idóneos, y reducción del sesgo}

Para apresar adecuadamente las competencias del bebé, y dada la complejidad a la que inicialmente nos hemos referido, resulta imprescindible desarrollar una planificación adecuada a partir de la concreción del problema o delimitación de objetivos, que, por supuesto, es previa al inicio de la recogida de datos.

Para reducir en gran medida los riesgos de error posterior conviene atender preferentemente a tres cuestiones que sostienen la consistencia del curso posterior del proceso:

2.1. Observación exploratoria, que es de carácter asistemático o casual, pero que tiene una gran importancia y debe prolongarse suficientemente. Son considerables las ventajas que se obtienen al llevar a cabo esta observación pasiva: a) El 
problema se acota adecuadamente; b) disminuye o se anula el sesgo de reactividad de los sujetos observados; c) mejora el nivel de entrenamiento del observador; y d) el bagaje de informaciones anecdóticas recogidas será muy útil para la toma de decisiones diversas (criterios de constancia intersesional, técnica de muestreo intrasesional, establecimiento o no de intervalos, número mínimo de sesiones, etc.) durante la observación activa.

2.2. Requisitos idóneos encaminados a facilitar la buena marcha del procedimiento, y que actúan como importante garantía para no incurrir en carencias o errores metodológicos que darían lugar indefectiblemente a registros falseados. Esencialmente son los siguientes:

a) Mantenimiento de la constancia intersesional: Con el fin de garantizar el máximo de homogeneidad entre las diferentes sesiones de observación es imprescindible que se haya elaborado una relación de los requisitos mínimos que permiten caracterizar el perfil de las sesiones de observación que se ajustan al objetivo propuesto. Los criterios pueden ser variados, siempre que resulten adecuados (días, lugar, hora, sujetos presentes, tiempo de actividad desempeñada, ausencia de interrupciones externas, etc.).

b) Mantenimiento de la constancia intrasesional: Evento inesperado o circunstancia sobrevenida en el transcurso de una sesión de observación, que ocasiona a su vez una ruptura del curso de la acción (actividad o línea argumental de la sesión). Se plantea en este caso si se puede aprovechar, contando con el principio de economía de esfuerzo, el registro correspondiente a la parte de la sesión previa, adoptándose como criterio convencional positivo el hecho de que en ella se cumpla la totalidad de las condiciones de constancia intersesional.

c) Tratamiento de las disrupciones temporales: Evento inesperado o circunstancia sobrevenida en el transcurso de una sesión de observación que ocasiona una interrupción de la misma, sin que se rompa el curso de la acción.

d) Especificación de las unidades de conducta: Unidad de conducta es la mínima información capaz de ser identificada, denominada, y que posee significado propio. Aquí se plantea el problema clave de la transducción y/o plasmación de la conducta del bebé al registro (Condon \& Ogston, 1967). En efecto, ¿podríamos siempre garantizar que si efectuamos una descripción de una conducta, o episodio, o escena del bebé, y la sistematizamos y codificamos convenientemente, podría ser decodificada y de nuevo "pasada a la acción descrita" sin pérdida relevante de información, o, lo que es aún más importante, sin distorsión? ¿Ofrecería más garantías un registro especialmente molar, o uno especialmente molecular?

d) Elaboración de un plan o agenda relativo a la sucesión de actividades a desarrollar a lo largo del proceso: Fase exploratoria, planteamiento del diseño del estudio y plan de muestreo observacional, elaboración del instrumento de observación, registro y simultánea comprobación del control de calidad del dato, desarrollo analítico del diseño, interpretación de resultados y elaboración del informe.

e) Identificación de la sesión de observación: Aparte de datos identificativos de fecha y hora, se incluirá información relativa a los cuatro niveles del contexto: a) Entorno físico (superficie, iluminación, mobiliario, etc.), b) actividad realiza- 
da o conductas del bebé, c) nivel social relativo al/a los sujeto/s observado/s (con indicación de sujetos presentes no observados, y de su constancia o variabilidad en las distintas sesiones), y d) información de carácter institucional u organizativo.

2.3. Reducción del sesgo. Son muy diversos los sesgos y dificultades que acechan al observador, pudiéndose solventar en su práctica totalidad con una planificación adecuada y un correcto adiestramiento de los observadores (Anguera, Blanco, Losada y Sánchez Algarra, 1999).

Los grandes grupos de sesgos son los siguientes:

a. La reactividad consiste en la alteración de la naturaleza espontánea de las conductas de los sujetos observados que se ocasiona precisamente cuando se aperciben de que están siendo observados. En nuestro caso es inexistente, debido a la corta edad de los bebés.

b. La expectancia surge en el observador en forma de previsiones y/o anticipaciones de conductas no contextualizadas, y, en ocasiones, ni siquiera percibidas.

c. En metodología observacional se contempla la denominada ecuación funcional $\mathrm{O}=\mathrm{P}+\mathrm{I}+\mathrm{Cp}-\mathrm{S} \quad(\mathrm{O}=$ Observación, $\mathrm{P}=$ Percepción, I=Interpretación, $\mathrm{C} p=$ Conocimiento previo, $\mathrm{S}=$ Sesgos), en donde la falta de equilibrio entre $\mathrm{P}, \mathrm{I}$ y Cp genera sesgos de carácter estructural.

d. Sesgos de carácter técnico, que son muy variados: Ángulo de mira incorrecto, planificación incorrecta del muestreo observacional, fallos de funcionamiento de medios técnicos, falta de sincronización entre los observadores de un equipo, etc.

\section{Instrumentos de observación}

La extraordinaria diversidad de conductas del bebé susceptibles de ser sistemáticamente observadas obliga a prescindir de instrumentos estándar y, por el contrario, dedicar el tiempo necesario a prepararlo ad hoc. Existen, como instrumentos básicos de la metodología observacional, el sistema de categorías y el formato de campo, que a su vez pueden combinarse entre sí, y a los que se incorpora, para determinadas cuestiones, la rating scale o escala de estimación. El sistema de categorías es de mayor rango por su imprescindible soporte teórico, mientras que los formatos de campo constituyen un instrumento más flexible especialmente adecuado en situaciones empíricas de elevada complejidad y de marco teórico endeble.

\subsection{Sistema de categorías}

Se trata de una construcción del observador correspondiente a un sistema nominal de registro que permite disponer de una especie de receptáculos o moldes elaborados a partir de un componente empírico (realidad) y de un marco teórico, y a los que se asignarán las conductas registradas. No sólo debe estudiarse la individualidad de cada una de las categorías, sino que es fundamental además la estructura de conjunto que forma el sistema.

El proceso es recurrente entre la realidad y el marco teórico. El punto de partida más recomendable es la elaboración del repertorio o lista de rasgos de conducta (realidad) de forma que cuente con presunción de exhaustividad, para lo cual se requerirán un buen número de sesiones de observación, y se fija alguna medida convencio- 
nal consistente en el establecimiento de un número mínimo de sesiones (tres, cuatro, cinco,...) sucesivas en las cuales no ocurra alguna nueva conducta distinta de las ya listadas. El paso siguiente consiste en proponer, a partir del marco conceptual, unos criterios que permitan realizar agrupaciones por afinidad entre los rasgos de conducta, y a las que se da una denominación provisional.

A continuación, volviendo a la realidad, se efectúa el visionado de nuevas sesiones, a la vez que se trata de asignar las conductas que nos interesan de acuerdo con el objetivo a las agrupaciones provisionales realizadas. Éste es el momento en que, a la luz del marco teórico, se analiza y revisa si existe un adecuado grado de homogeneidad entre las conductas, procediéndose, según sea el caso, a desglosar alguna de las agrupaciones, o a modificar otras, etc., de forma que se preserve una diferenciación conceptual entre las categorías provisionales que se van fraguando, una posibilidad de asignación de todas las conductas de interés a alguna de tales categorías, y, además, que exista homogeneidad entre las conductas asignadas a estas categorías provisionales. Una vez realizadas estas modificaciones, de nuevo se procede a visionar nuevas sesiones, asignando las conductas a la nueva propuesta de categorías, y así se va repitiendo el proceso de forma iterativa hasta que el conjunto de las categorías configura un sistema exhaustivo dentro del área o situación observada y mutuamente excluyente en cada una de las dimensiones o niveles implicados (Anguera, 1991).

La exhaustividad se refiere a que cualquier comportamiento del ámbito considerado como objeto de estudio (que habrá sido seleccionado y muestreado del repertorio conductual del sujeto) puede asignarse a una de las categorías. Y la mútua exclusividad significa el no solapamiento de las categorías que componen un sistema, por lo que a cada comportamiento se le asignaría una y sólo una categoría. Sin embargo, y desde el punto de vista de los niveles que interesen, puede no ser posible -ni incluso conveniente en ocasiones-, ya que en muchas ocasiones interesa contemplar varios niveles de respuesta co-ocurrentes, por lo que se crearían categorías múltiples que abarquen todas las posibles combinaciones entre las iniciales.

Las categorías tienen que definirse de forma que se contemplen todos sus matices, así como acompañarse de ejemplos y contraejemplos -que pueden adoptar el formato gráfico o de fotografía (Losada, 1999)- para que su especificación sea mayor.

Cuando no se produce ocurrencia de conducta, deberá registrarse mediante la categoría formal $\varnothing$ (conjunto vacío).

Dado que la elección de unas categorías u otras no es única en absoluto, sino que en buena medida depende de quién las elabore, los sistemas de categorías relativos a una determinada situación o comportamientos serán equivalentes si durante el proceso de categorización se adoptan los mismos criterios, pero se trata de una equivalencia en su conjunto, no categoría por categoría, sino el conjunto formado por todos los núcleos categoriales. En consecuencia, tiene sentido la comparabilidad de dos o más sistemas de categorías, lo cual puede dar lugar a cuestiones interesantes.

El lento proceso de construcción de un sistema de categorías, que se va optimizando hasta que se adapta adecuadamente a la situación para la cual fue elaborado, puede dar lugar frecuentemente a la consideración de una falsa estabilidad, dado que su carácter de "instrumento acabado" es sólo relativo, puesto que, especialmente en estudios que se prolongan considerablemente a lo largo del tiempo, la propia evolución de la realidad puede generar un progresivo desajuste con las categorías estudiadas. 
Finalmente, conviene tener en cuenta la posibilidad de estructurar jerárquicamente un sistema de categorías, desde una mayor molaridad a una mayor molecularidad. Esta opción de sistema escalonado de categorías ofrece indudables posibilidades de desarrollo analítico a nivel de diseño.

\subsection{Formatos de campo}

Su origen se remonta al de una vieja técnica de registro (Weick, 1968) que ha sido recuperada (Anguera, 1979), y ha ido ganando consistencia y adquiriendo los atributos necesarios desde que se empezó a utilizar como tal en España (Lareo, 1984), permitiendo que en la actualidad se la pueda considerar con el rango de instrumento de observación, una vez ha sido optimizada. A lo largo de los últimos años, su uso se ha incrementado de forma espectacular.

Su elaboración implica los siguientes pasos:

1) Establecimiento de criterios o ejes del instrumento, fijados en función de los objetivos del estudio (por ejemplo, en actividad física realizada en una escuela maternal, los posibles criterios serían la ubicación, actividad, interactividad, contacto con objetos, etc.). Es posible que alguno de los criterios se desglose jerárquicamente en otros.

2) Listado de conductas/situaciones (lista no cerrada) correspondientes a cada uno de los criterios, anotadas a partir de la información que proporcionó la fase exploratoria del estudio.

3) Asignación de un sistema de codificación decimal a cada una de las conductas/situaciones anotadas que deriven de cada uno de los criterios, lo cual permite desplegar cualquiera de ellos en un sistema jerárquico de orden inferior. En función de la complejidad del caso o rango de molecularidad deseado, se puede tratar de sistemas de doble, triple, etc., código.

4) Elaboración de la lista de configuraciones. La configuración es la unidad básica en el registro de formatos de campo, y consiste en el encadenado de códigos correspondientes a conductas simultáneas o concurrentes, el cual permitirá un registro exhaustivo del flujo de conducta, y una enorme facilitación para posteriores análisis de datos.

Las configuraciones se rigen por los criterios sincrónico y diacrónico: Sincrónico, porque todos los códigos de cada configuración corresponden a conductas -una de cada criterio- simultáneas, de forma que, al modificarse uno o más códigos de una configuración, ello da lugar a la siguiente. El criterio diacrónico se apoya, precisamente, en esta sucesión de configuraciones.

Si cotejamos los dos tipos de instrumentos -sistema de categorías (SC) y formatos de campo (FC)-, las principales diferencias entre ellos se reflejan en la tabla siguiente. 
Tabla 1. Comparación entre sistemas de categorías y formatos de campo.

\begin{tabular}{llll}
\hline Criterios & Sistema de categorías & Formato de campo & Ventaja a favor de... \\
\hline Estructura & Sistema cerrado & Sistema abierto & FC \\
\hline Relación con teoría & $\begin{array}{l}\text { Marco teórico } \\
\text { imprescindible }\end{array}$ & $\begin{array}{l}\text { Marco teórico } \\
\text { recomendable, } \\
\text { pero no imprescindible }\end{array}$ & SC \\
\hline Dimensionalidad & Unidimensional & Multidimensional & FC \\
\hline Codificación & De código único & De código múltiple & FC \\
\hline Flexibilidad & Sistema rígido & Sistema autorregulable & FC \\
\hline
\end{tabular}

\subsection{Combinación de formatos de campo y sistemas de categorías}

Con relativa frecuencia es recomendable anidar uno o más sistemas de categorías en uno o más de los criterios de los formatos de campo, respectivamente, de manera que la relación de conductas/situaciones que corresponden a este(estos) criterio(s) serían las categorías que conforman el sistema, y, por tanto, son exhaustivas y mutuamente excluyentes a nivel intra-criterio del formato de campo.

Con ello se logra mantener la mayor flexibilidad de los formatos de campo, a la vez que, siempre que el objeto estudiado y/o la situación lo recomiendan, se incorporan unas categorías rígidas como desarrollo del criterio que lo precise (en muchos casos por exigencias del reglamento del deporte respectivo).

\subsection{Rating scales o escalas de apreciación}

Este instrumento de observación, que corresponde a un sistema dimensional de registro, tiene un uso restringido debido al necesario requisito de ordenación de un atributo o dimensión, que no siempre es posible ni fácil.

Precisamente es en el ámbito de captación de las conductas del bebé donde alcanza su máxima potencialidad, debido a la posibilidad de reflejar el desarrollo progresivo de un determinado sector evolutivo (Cambrodí y Sastre, 1993).

Las rating scales permiten efectuar un primer despliegue de las diferentes áreas o subáreas en que pretenden efectuar una medida del comportamiento producido. Para cada una de ellas debe prepararse una serie de descripción de ejecuciones que estén graduadas en cuanto a complejidad, grado de dificultad, etc., indicando la asignación de puntuaciones.

A pesar de que les acompañe una correcta definición a cada una de las estimaciones, hay que contar con la existencia de un riesgo de subjetividad en la mayoría de los casos.

\section{Registro}

Registro es la transcripción de la representación de la realidad por parte del observador mediante la utilización de códigos determinados y que se materializa en un soporte físico que garantiza su prevalencia (adaptado de Anguera, Behar, Blanco, Carreras, Losada, Quera y Riba, 1993). 
Debido a que los datos posibles y su producción en la realidad presentan una variabilidad casi infinita, los tipos o técnicas de registro desarrollados son igualmente innumerables, obedeciendo a la naturalidad y espontaneidad de la conducta observada y al hecho de que la metodología observacional sea una estrategia altamente flexible.

Son múltiples las preguntas que nos podemos formular en la observación de bebés: ¿Qué registrar?, ¿dónde registrar?, ¿cómo registrar?, ¿cuál es la métrica del registro?, a las cuales debemos dar respuesta.

\section{1. ¿Qué registrar?}

En el ámbito infantil temprano deberemos registrar aquellos sectores del comportamiento que podamos percibir y nos permitan objetivar (y posteriormente cuantificar) su estudio. Indudablemente su alcance se halla únicamente restringido por la perceptibilidad, y, en consecuencia, por la posibilidad de captar la ocurrencia de conductas mediante nuestros órganos sensoriales (especialmente visuales y auditivos), en la forma más primitiva, o mediante grabación y almacenamiento de la información.

Todos los niveles clásicos de respuesta caben en el estudio del comportamiento infantil. Siendo conscientes de sus limitaciones, sugerimos la clasificación de niveles de respuesta de Weick (1968), que corresponden al "contenido" de la conducta a observar, y en el bien entendido de que nos puede interesar uno de ellos o varios simultáneamente:

a) La conducta no verbal se refiere a las expresiones motoras que pueden originarse en distintas partes de su cuerpo. Se trata quizá del área más activa de las recientes investigaciones en metodología observacional, mostrándose la relevancia de los movimientos del cuerpo. Además, la conducta no verbal es extremadamente sutil para el registro, siempre que el observador esté entrenado y adiestrado (Anguera, Blanco, Losada y Sánchez-Algarra, 1999) y sea sensible a sus manifestaciones. La propuesta inicial, efectuada por Weick (1968), desglosaba la conducta no verbal en expresiones faciales, intercambios de mirada y movimientos corporales. No obstante, entendemos que se incurre en dos problemas metodológicos, lo cual nos ha llevado a introducir una modulación. Por una parte, entre expresiones faciales e intercambios de mirada no se cumple la mutua exclusividad, dado que la segunda constituiría un subconjunto de la primera, y los movimientos corporales no hacen posible que sea efectiva la condición de exhaustividad con el resto, dado que únicamente se contemplaría la conducta gestual (que es dinámica), pero no la postural (que es estática). Teniendo en cuenta que, desde un criterio topográfico del ser humano, siempre se produce alternancia entre conducta gestual (conducta dinámica entre dos conductas estáticas) y conducta postural (conducta estática entre dos conductas dinámicas), la modulación introducida consistiría en contemplar, como modalidades de la conducta no verbal, expresiones faciales, conducta gestual y conducta postural. Y, como apunte último, entendemos que esta última propuesta es sin perjuicio de que, en un futuro no lejano, se entienda dicotomizada en conducta gestual y postural, pues aunque cada vez en mayor medida las expresiones faciales se consideran como la expresión privilegiada de los estados emocionales del deportista, es igualmente cierto que pueden reducirse a las otras dos modalidades de conducta. 
b) La conducta espacial o proxémica presenta dos vertientes: Una es de carácter estático, y se refiere a la elección de lugar en un espacio, como el bebé que deambula sobre la alfombra de una habitación y elige "su" zona. La segunda vertiente es mucho más relevante en el ámbito infantil, y comprende el conjunto de los desplazamientos de un bebé, realización de trayectorias, ocupación del espacio, etc.

c) La conducta vocal o extralingüística estudia los diversos aspectos de interés en la vocalización, sin que interese en absoluto el contenido del mensaje. A lo largo de los años, la incidencia de nuevas tecnologías ha revolucionado este tipo de conducta no verbal. Así, desarrollos informáticos o el actual sonógrafo permiten efectuar una descomposición espectral de la voz, a la vez que se calcula la energía producida en el instante de su emisión y se detecta el formante, identificador de cualquier individuo. Las aplicaciones en el ámbito infantil son muy interesantes, especialmente en el ámbito del desa-rrollo del lenguaje.

d) La conducta verbal o lingüística, al contrario que la vocal o extralingüística, se refiere al contenido del mensaje, y es la que tiene una aplicación más limitada en la observación de bebés, debido a su corta edad. Por este motivo, nos debemos remitir al análisis del texto resultante de su transcripción.

Nuestro objeto de estudio es el que nos delimitará qué conductas debemos registrar. Otra cuestión distinta es que no esté bien acotado, y por este motivo convendrá que diferenciemos perfectamente las fases de observación pasiva y observación activa.

\section{2. ¿Dónde registrar?}

En la época clásica de la metodología observacional, lo habitual era el registro in situ e in vivo, lo cual implicaba una serie de riesgos, entre los cuales destacaba la distorsión producida por la imposibilidad material de registrar todas las conductas que interesaban, y especialmente en comportamientos fugaces, como suelen ser todos los de bebés, así como una habitual alteración de la información registrada si no se registraba inmediatamente después de producirse, además de un elevado riesgo de reactividad de los individuos observados (Behar y Riba, 1993). Por tanto, concurrían diferentes tipos de sesgos y aumentaban los errores en el registro.

Todo estudio científico pretende reducir y eliminar los errores, y este interés aumenta, si cabe, en la observación de comportamientos infantiles, debido a que las situaciones de observación son complejas, existe una movilidad intrínseca, la mayoría de las conductas son fugaces, los estudios son habitualmente prolongados, etc. Es cierto que después se someterá el registro a un control de calidad del dato, pero previamente debe efectuarse un planteamiento tendente a reducir riesgos de error.

En las últimas décadas, el impresionante avance tecnológico que se ha producido ha facilitado en gran medida el rigor y la precisión en el registro. En la actualidad, en la práctica totalidad de los casos se puede disponer de la grabación de las sesiones de observación, motivo por el cual la operación metodológica de registro se disocia en las de grabación-visionado(s)-registro a partir de la grabación, pudiéndonos ayudar mediante instrumentos mecánicos o, especialmente, tecnológicos (Losada, 1993). 


\section{3. ¿Cómo registrar?}

La fase empírica de la observación se inicia desde el momento en que el observador empieza a acumular y clasificar información, con lo que adquiere unos datos provenientes de un volcado de la realidad, y que deberá sistematizar progresivamente, pudiéndolo hacer a lo largo de una gradación con muchos eslabones intermedios, los cuales suelen sucederse entre sí, al menos parcialmente, a medida que avanza el conocimiento del observador acerca de las conductas estudiadas y se acrecienta su rodaje específico.

Son prácticamente incontables las modalidades de registro existentes o que se pueden crear, y en su elección será relevante la consideración de los objetivos y de los contextos en que se ubique el estudio (así, registro continuo vs. intermitente, registro de conductas más o menos molarizadas, etc.). De forma tradicional, se han establecido cuatro grandes sistemas de registro (y codificación, a la que nos referiremos en el apartado siguiente) (Blanco y Anguera, 1991; Blanco, 1997): Sistemas verbales, nominales, dimensionales y estructurales.

El nivel de sistematización (o grado de control externo) de un registro constituye el criterio más relevante para diferenciar diversas modalidades de registro, teniendo en cuenta que matizaremos las diferencias entre los principales tipos, a sabiendas de que nos referiremos únicamente al prototipo de cada uno de los grados de sistematización: Registro narrativo (que se corresponde con los sistemas verbales), registro descriptivo (ambos son no sistematizados), registro semi-sistematizado y registro sistematizado (a medida que aumenta el grado de sistematización se corresponden con los sistemas nominales), y posteriormente, en sucesivos apartados, haremos alguna referencia a los sistemas dimensionales y estructurales.

\begin{tabular}{llll}
\hline $\begin{array}{l}\text { Observación } \\
\text { Pasiva }\end{array}$ & Observación activa & \\
Registro & Registro & Registro & $\begin{array}{l}\text { Registro } \\
\text { sistematizado }\end{array}$ \\
Narrativo & descriptivo & semi-sistematizado & \\
& & &
\end{tabular}

Figura 1. Modalidades de registro en la observación del comportamiento deportivo.

\subsection{Métrica del registro}

Actualmente se halla fuera de toda duda que, pese al carácter fundamentalmente cualitativo de los registros observacionales, es imprescindible no sólo la codificación, o transformación de estos datos de forma que sean susceptibles de un tratamiento cuantitativo, sino la obtención de diversos tipos de medida, que son: Frecuencia, orden y duración (además de la intensidad, cuando es factible, y de otros de menor relevancia), como parámetros básicos, además de los secundarios, que se derivan de ellos.

Estos tres parámetros básicos guardan entre sí una relación progresiva de inclusión, y constituyen un punto de referencia para otras cuestiones, como tipos de 
datos, índices de acuerdo entre observadores, etc. Veamos una noción escueta de cada uno de ellos.

La frecuencia (F) es el número de ocurrencias de determinada categoría o código de formatos de campo en el transcurso de un período de tiempo previamente fijado, tanto si se trata de una sesión (tiempo ininterrumpido de observación que no tiene que coincidir con días), de un intervalo (período regular limitado por unidades convencionales de tiempo), u otra unidad. Su capacidad informativa es sumamente baja. Medidas derivadas de la frecuencia son: Tasa o razón de ocurrencia, frecuencia modificada y frecuencia modificada de Sanson-Fisher.

El orden $(\mathrm{O})$ aporta una información fundamental en el registro, dado que facilita un posterior estudio de la secuencialidad de la conducta, y ofrece, a su vez, nuevas e interesantes perspectivas de análisis que no serían accesibles con planteamientos no secuenciales. Lo que se registra es la sucesión de códigos correspondientes a las categorías o a las configuraciones de formatos de campo.

La duración (D) registra unidades convencionales de tiempo que abarca cada ocurrencia de una determinada conducta.

\section{Control de la calidad del dato}

Una vez realizada la recogida de datos, el observador debe tener la garantía necesaria sobre su calidad, y el más básico de los requisitos de control es precisamente lo que tradicionalmente se denominó fiabilidad del registro observacional.

Al abordar esta temática se utilizan términos diversos con matices e incluso significado distinto (Blanco, 1989, 1993; Bakeman \& Gottman, 1989); de aquí que deba quedar claro que se ha producido una reconceptualización desde el uso indiscriminado del término fiabilidad, dado que no contamos con lo que sería una medida precisa (al no disponer de instrumento estándar, sino elaborado ad hoc).

En la observación de bebés, aunque en menor medida que en sujetos adultos, existen diversos factores que están incidiendo de forma diversa sobre las conductas que se ejecutan, y de aquí que nos preguntemos si los valores observados son interpretables, o si, por el contrario, son el resultado de fluctuaciones aleatorias introducidas por la misma medida. De aquí que Blanco (1997) desarrolle tres formas de entender la fiabilidad de los datos observacionales: a) Coeficientes de concordancia entre dos observadores que, registrando de forma independiente, codifican las conductas mediante un mismo instrumento de observación; b) coeficientes de acuerdo, resueltos mediante la correlación; y c) aplicación de la teoría de la generalizabilidad, cuando interesa integrar diferentes fuentes de variación (observadores distintos, diversas ocasiones, varios instrumentos, tipos variados de registro, ocasiones diversas, etc.) en una estructura global.

Además de las formas cuantitativas de control de la calidad del dato, cada vez la concordancia consensuada cuenta con mayor protagonismo en metodología observacional. Se trata de lograr el acuerdo entre los observadores antes del registro (y no después, como corresponde en los diferentes coeficientes a los que da lugar la forma cuantitativa), lo cual puede conseguirse siempre que se disponga de la grabación de la conducta (mediante magnetófono, si sólo interesa conducta vocal y/o verbal, o mediante video, en cualquier caso) y los observadores discuten entre sí a qué categoría se asigna cada una de las unidades de conducta. Presenta ventajas evidentes, y a 
la obtención de un registro único hay que añadirle un importante fortalecimiento del instrumento de observación, ya que quedan mejor perfiladas sus definiciones y los matices que deban añadirse. No obstante, no podemos olvidar los inconvenientes que supone el hecho de que un determinado observador "pase" de asignar una conducta a una determinada categoría o código de formatos de campo por confiar en el prestigio o elevada competencia de otro(s) observador(es), aceptando sus propuestas; o, por el contrario, que afloren problemas de dinámica social y se proyecten a una habitual dificultad para lograr el consenso entre los observadores.

\section{Diseños observacionales}

La metodología observacional adoleció tradicionalmente de líneas de investigación en las cuales se pusieran a prueba las múltiples posibilidades de análisis de sus datos. Probablemente, el principal motivo se halla en la superficialidad con que se obtenían tales datos, y, por consiguiente, en su carácter inconsistente.

En la actualidad, sin embargo, casi al final de la década de los noventa, se ha avanzado en la configuración de los principales diseños observacionales, los cuales son de disposición no estándar en coherencia con el carácter sumamente flexible de la metodología observacional, y si además tenemos en cuenta una sustancial mejora que en la mayoría de los casos se pone en práctica en el proceso de sistematización y optimización de los datos, es lógico que hayan surgido nuevas propuestas en lo que se refiere al análisis de datos.

Por supuesto, se pueden establecer criterios muy diversos en este punto de encrucijada. Desde hace más de una década estamos trabajando con un planteamiento (Anguera, 1985, 1995) en que se cruzan la dicotomía idiográfico (unidad) / nomotético (pluralidad) y la relativa a un registro puntual / seguimiento, lo cual facilita el deslinde de las direcciones básicas de análisis de datos observacionales (Figura 2).

\begin{tabular}{|c|c|}
\hline \multicolumn{2}{|c|}{ Idiográfico } \\
\hline II & I DISEÑOS DIACRÓNICOS \\
\hline Puntual & Seguimiento \\
\hline III DISEÑOS SINCRÓNICOS & IV DISEÑOS MIXTOS O LAG-LOG \\
\hline
\end{tabular}

Figura 2. Diseños evaluativos de baja intensidad: Tipos básicos.

\subsection{Cuadrante I: Diseños diacrónicos}

El seguimiento en un estudio idiográfico (cuadrante I) constituye, por esencia, una situación en la cual se consigue focalizar toda la atención en un bebé o nivel de respuesta (como su conducta vocal), o unidad mínima de varios sujetos que funcione como unidad (como la interacción diádica madre-hijo). 
Es prácticamente imposible establecer una sistemática que incluya todas las posibilidades de diseños diacrónicos en evaluación de intervenciones por la propia concepción expresada del término diseño y por la gran casuística existente. Pero, mediante una serie de tomas de decisiones relativas a criterios básicos, complementarios y moduladores (Tabla 2), se puede elaborar una sistemática adecuada para este tipo de diseños, que, como todos los de baja intensidad interventiva, gozan de elevada flexibilidad, reduciéndose a pautas básicas a seguir.

Estos tres tipos de criterios actúan de forma progresiva, motivo por el cual se sientan los fundamentos del diseño en los criterios básicos, que se refieren únicamente a los dos indicados anteriormente (cuadrante I, por ser idiográfico y de seguimiento), a lo cual se añade el parámetro primario de registro, que, a su vez, permite diferenciar entre seguimiento extensivo y seguimiento intensivo, según que, respectivamente, sea la frecuencia o el orden (al cual se podría añadir la duración). Una vez delimitados los criterios básicos, los complementarios se refieren esencialmente al instrumento observacional utilizado en la recogida de datos, así como, en su caso, a la posible codificación binaria. Finalmente, los criterios moduladores están basados en los dos grandes niveles del muestreo observacional (intersesional e intrasesional); respecto a la incidencia del plan de muestreo intersesional, habrá que tener en cuenta los múltiples matices que se derivan, pues, por ejemplo, no es lo mismo que un bebé se observe intensivamente durante un fin de semana que a lo largo de tres meses una hora diaria.

Tabla 2. Toma de decisiones sobre los diseños diacrónicos.

\begin{tabular}{|c|c|c|c|c|c|}
\hline Modalidad & Tipo & $\begin{array}{l}\text { Análisis } \\
\text { de datos }\end{array}$ & $\begin{array}{l}\text { Criterios } \\
\text { básicos }\end{array}$ & $\begin{array}{l}\text { Criterios } \\
\text { complementarios }\end{array}$ & $\begin{array}{l}\text { Criterios } \\
\text { moduladores }\end{array}$ \\
\hline \multirow[t]{2}{*}{ EXTENSIVO } & $\begin{array}{l}\text { Panel } \\
\text { Tendencia } \\
\text { Series } \\
\text { temporales }\end{array}$ & $\begin{array}{l}\text { Análisis } \\
\text { de panel } \\
\text { Análisis de } \\
\text { regresión } \\
\text { Análisis de } \\
\text { series de } \\
\text { tiempo }\end{array}$ & $\begin{array}{l}\text { Idiográfico } \\
\text { Seguimiento } \\
\text { Frecuencia }\end{array}$ & $\begin{array}{l}\text { - Codificación } \\
\text { Binaria } \\
\text { - Sistema de } \\
\text { categorías } \\
\text { - Formatos } \\
\text { de campo } \\
\text { - Escala de } \\
\text { apreciación }\end{array}$ & $\begin{array}{l}\text { Muestreo intersesional: } \\
\text { Período recogida } \\
\text { de datos } \\
\text { Periodicidad } \\
\text { № sesiones } \\
\text { Criterios inicio } \\
\text { Criterios } \\
\text { finalización }\end{array}$ \\
\hline & & & & & $\begin{array}{l}\text { Muestreo intrasesional: } \\
\text { Puntos de tiempo } \\
\text { Intervalos totales } \\
\text { Intervalos parciales }\end{array}$ \\
\hline INTENSIVO & $\begin{array}{l}\text { Secuencial } \\
\text { Coordenadas } \\
\text { polares }\end{array}$ & $\begin{array}{l}\text { Análisis } \\
\text { secuencial } \\
\text { Análisis de } \\
\text { Coordenadas } \\
\text { polares }\end{array}$ & $\begin{array}{l}\text { Idiográfico } \\
\text { Seguimiento } \\
\text { Orden (al } \\
\text { que se puede } \\
\text { añadir la } \\
\text { duración) }\end{array}$ & $\begin{array}{l}\text { - Sistema de } \\
\text { categorías } \\
\text { - Formatos } \\
\text { de campo }\end{array}$ & $\begin{array}{l}\text { Muestreo intersesional: } \\
\text { Período recogida } \\
\text { de datos } \\
\text { Periodicidad } \\
\text { № sesiones } \\
\text { Criterios inicio } \\
\text { № datos registrado } \\
\text { Muestreo intrasesional: } \\
\text { Registro continuo }\end{array}$ \\
\hline
\end{tabular}




\subsubsection{Diseños diacrónicos extensivos}

Los diseños diacrónicos extensivos se diferencian entre sí en función del número de puntos de anclaje, es decir, momentos de recogida de información.

\section{Diseños de panel}

Corresponde al caso de un seguimiento totalmente exiguo, con sólo dos puntos en el diseño típico (o tres, en algunas variantes) de recogida de datos. Nos enfrentamos a la situación más desfavorable, debido a la escasez de información, que hace muy endeble el anclaje empírico. Por tanto, únicamente se pueden detectar los cambios "de malla ancha" en la frecuencia de conductas del bebé entre el momento de tiempo $\mathrm{t}$ y el $\mathrm{t}+1$.

Ofrece la posibilidad de análisis del cambio a nivel individual y colectivo.

\section{Diseño de tendencia}

El diseño de tendencia permite realizar inferencias acerca de la eficacia o impacto de un programa de intervención en el bebé, observando los cambios producidos antes o después de que se inicie un determinado tratamiento. Puede hallarse de varias formas, aunque la más habitual es el cálculo de la recta de regresión (Kratochwill, 1989), para lo cual no es problema que los datos sean de naturaleza categórica (Kritzer, 1986); en caso de que los distintos puntos no se ajusten a una tendencia li-neal, habrá que buscar otros tipos de ajuste (logarítmico, exponencial, etc.).

A diferencia del diseño de panel, la búsqueda de la relación funcional permite realizar predicciones, con el consiguiente riesgo de error. De ahí que, a pesar de que en los requisitos de mínimos, en el diseño de tendencia hay cuantitativamente un solo punto más de recogida de información, sin embargo, cualitativamente es mucho mejor.

\section{Diseño de series temporales}

En procesos de duración prolongada no es infrecuente plantear un diseño de series temporales. Una serie temporal es una secuencia cronológicamente ordenada de valores de medición, que en diseños diacrónicos extensivos son frecuencias, a lo largo de cincuenta o más puntos equidistantes de tiempo. Lo que se pretende es la búsqueda de regularidades del comportamiento del bebé en el tiempo, así como el pronóstico, predicción, previsión o vaticinio de su comportamiento más probable en el futuro.

La característica fundamental para juzgar sobre la consistencia de una serie temporal es la invariabilidad en la forma de recogida de los datos a través del tiempo. Como dice Aguirre Jaime (1994), debe tenerse presente que una serie temporal recopilada con un método primitivo y sesgado, pero inmutable a través de los años, es mucho más consistente en relación con una serie recopilada a través de métodos más eficientes, pero con cambios en el transcurso del tiempo.

La situación más habitual es la de plantear un análisis de series de tiempo, que no deja de ser un caso particular de diseños intrasujeto, en donde los datos se caracterizan esencialmente por su dependencia serial, y el principal interés se centra en el estudio del modelo ARIMA (Auto Regressive Moving Average), así como el análisis de sus componentes. Tradicionalmente era poco usual su cálculo a par- 
tir de variables categóricas, pero en la actualidad (Bakeman y Gottman, 1989; Aguirre Jaime, 1994; Murillo, 1994) se puede efectuar sin problema.

\subsubsection{Diseños diacrónicos intensivos}

Los diseños diacrónicos intensivos se diferencian entre sí en función de la naturaleza de la relación que se halla entre las categorías o códigos de formatos de campo y su respectiva representación en forma de mapa.

\section{Diseño secuencial}

Las características que lo definen (idiográfico y seguimiento) son especialmente idóneas para la detección de patrones de conducta.

La técnica analítica que lo posibilita está perfectamente estudiada en la actualidad, y principalmente se utilizan dos programas informáticos, que parten, a su vez, de un planteamiento diferente: SDIS-GSEQ (Bakeman \& Gottman, 1997; Bakeman \& Quera, 1992, 1996; Bakeman, Quera, McArthur \& Robinson, 1997; Quera, 1993; Escudero, 1995; Losada, 1995; Morales, 1996) y THÈME (Magnusson, 1993, 1996, 2000; Magnusson \& Beaudichon, 1997; Montagner, Magnusson, Casagrande, Restoin, Bel, Nhuyen, Paul, Ruiz, Delcourt, Gaufeier \& Epoulet, 1990; Pastor y Sastre, 1999).

El SDIS-GSEQ se apoya en una técnica analítica que fue desarrollada por Bakeman (Bakeman, 1978; Bakeman y Gottman, 1989), y por Sackett (1980, 1987), a partir de los antecedentes que se hallan en los trabajos de Bakeman \& Dabbs (1976). A su vez, caben dos perspectivas: Prospectiva (contemplando el sentido "hacia delante", tal cual se produce la ocurrencia de conducta) y retrospectiva (en sentido "hacia atrás"), que nos proporciona una imagen especular del patrón de conducta, que permite contemplar las dos vertientes del diseño diacrónico intensivo secuencial.

El THÈME es un programa informático desarrollado por Magnusson (1993, 1996), que permite detectar patrones de conducta desde una lógica de tiempo real, tanto a nivel inter como intraindividual.

\section{Diseño de coordenadas polares}

Mientras que el diseño secuencial es unidireccional (sea en sentido prospectivo o retrospectivo), en el diseño de coordenadas polares se integran ambas. El objetivo es ofrecer un mapa en donde se muestren todas las interrelaciones existentes entre los códigos de categorías o de configuraciones de formatos de campo.

La técnica analítica que se utiliza es la de coordenadas polares, que fue propuesta por Sackett (1980). El diseño de coordenadas polares se enmarca en el grupo de diseños diacrónicos intensivos por ser idiográfico el planteamiento de la evaluación y realizarse un seguimiento intrasesional en el registro mediante el parámetro orden. Dentro de este grupo, se trata del planteamiento más complejo, que va encaminado a la obtención de una representación vectorial de la compleja red de interrelaciones que se establecen entre las distintas categorías o códigos de formato de campo. A su vez, y debido a la integración que se efectúa de las perspectivas diacrónica y sincrónica, que generarían gran volumen de resultados parciales, actúa también, de forma colateral, como técnica reductora de datos. Expresado de otra forma, los valo- 
res obtenidos en forma de probabilidades condicionales pasarán, a su vez, a constituirse en datos de un proceso de reducción que desembocará en la consecución de unos pocos parámetros indicativos sobre los cuales recaerá el peso interpretativo de los vectores que materializan el mapa interrelacional que se elabora.

\subsection{Cuadrante II}

El cuadrante II se caracteriza por ser idiográfico y puntual: Una recogida de datos puntual y a partir de una sola unidad (sea un bebé, un pequeño grupo de bebés o bebés-adultos que actúan conjuntamente, o bien un solo nivel de respuesta en un bebé o en un pequeño grupo) no es capaz de proporcionar información mínimamente consistente que garantice la cientificidad del estudio. En consecuencia, se trata del único cuadrante que no puede sustentar un diseño $y$, en consecuencia, no ofrece datos válidos para un posterior análisis.

\subsection{Cuadrante III: Diseños sincrónicos}

Cada vez son más frecuentes las situaciones en las que es necesario evaluar la interrelación entre diversas unidades (sean varios bebés o diversos niveles de respuesta de un mismo bebé) a partir de un registro de naturaleza puntual, y que habrán dado lugar, lógicamente, a diversos sistemas de categorías. La situación más habitual que se plantea es la de una situación en donde un comportamiento o actividad del bebé se taxonomiza simultáneamente desde diversos criterios, con lo que se obtienen datos pertenecientes de forma puntual a una sesión (e incluso a varias) que responden a los diversos sistemas de categorías o formatos de campo correspondientes a cada uno de los criterios establecidos, que pueden cruzarse y dar lugar a una tabla de contingencia.

Para establecer una sistemática de los diseños sincrónicos, igual como hicimos con los diseños diacrónicos, planteamos una serie de tomas de decisiones relativas a criterios básicos, complementarios y moduladores (Tabla 3), dejando claro que se trata de diseños que gozan de elevada flexibilidad, reduciéndose a pautas básicas a seguir.

Tabla 3. Toma de decisiones sobre los diseños sincrónicos.

\begin{tabular}{|c|c|c|c|c|}
\hline Modalidad & $\begin{array}{l}\text { Análisis } \\
\text { de datos }\end{array}$ & $\begin{array}{l}\text { Criterios } \\
\text { básicos }\end{array}$ & $\begin{array}{l}\text { Criterios } \\
\text { complementarios }\end{array}$ & $\begin{array}{l}\text { Criterios } \\
\text { moduladores }\end{array}$ \\
\hline \multirow[t]{2}{*}{ SIMÉTRICO } & \multirow{2}{*}{$\begin{array}{l}\text { Análisis } \\
\text { log-lineal }\end{array}$} & \multirow{2}{*}{$\begin{array}{l}\text { Nomotético } \\
\text { Puntual } \\
\text { Frecuencia } \\
\text { Rel. Asociativa }\end{array}$} & \multirow{2}{*}{$\begin{array}{l}\text { Codificación binaria } \\
\text { Sistema de categorías } \\
\text { Formatos de campo }\end{array}$} & - Muestreo intersesional \\
\hline & & & & $\begin{array}{l}\text { - Muestreo intrasesional } \\
\text { Registro continuo } \\
\text { Puntos de tiempo } \\
\text { Intervalos }\end{array}$ \\
\hline \multirow[t]{2}{*}{ ASIMÉTRICO } & \multirow{2}{*}{$\begin{array}{l}\text { Análisis } \\
\text { logit }\end{array}$} & \multirow{2}{*}{$\begin{array}{l}\text { Nomotético } \\
\text { Puntual } \\
\text { Frecuencia } \\
\text { Rel. causalidad }\end{array}$} & \multirow{2}{*}{$\begin{array}{l}\text { Codificación binaria } \\
\text { Sistema de categorías } \\
\text { Formatos de campo } \\
\text { Viabilidad de relaciones } \\
\text { de precedencia } \\
\text { de conducta }\end{array}$} & - Muestreo intersesional \\
\hline & & & & $\begin{array}{l}\text { - Muestreo intrasesional } \\
\text { Registro continuo } \\
\text { Puntos de tiempo } \\
\text { Intervalos }\end{array}$ \\
\hline
\end{tabular}


Estos tres tipos de criterios actúan de forma progresiva, motivo por el cual los diseños sincrónicos se asientan sobre los criterios que quedan definidos por el cuadrante en que nos hallamos (nomotético y registro puntual), a lo cual se añade el parámetro primario de registro, que es la frecuencia.

En los dos diseños sincrónicos, la estrategia de registro previa más adecuada sería la de eventos clasificados de forma cruzada (Bakeman y Gottman, 1989), de forma que no se requiere ningún tipo de continuidad entre eventos sucesivos, sino que resulta al ser captadas secuencias conductuales que son clasificadas desde varias dimensiones, aplicándose a ellas de forma concurrente los diversos instrumentos elaborados (sistemas de categorías o formatos de campo).

Hay dos tipos de diseños sincrónicos: Simétricos y asimétricos.

\section{Diseños sincrónicos simétricos}

Se caracterizan por la búsqueda de relaciones de asociación entre la pluralidad de elementos que disponemos. En cuanto al análisis de datos pertinente, gracias a los esfuerzos pioneros de Bishop (1969), Fienberg (1977) y Goodman (1970, 1971), y posteriormente de Kennedy (1983), entre otros, se ha desarrollado el análisis de tablas de contingencia mediante modelos lineales logarítmicos, o análisis log-linear, planteado en investigaciones de naturaleza simétrica (sólo interesa la presencia o ausencia de asociación entre las variables, sin direccionalidad u orden causal entre ellas).

\section{Diseños sincrónicos asimétricos}

Los diseños sincrónicos asimétricos introducen el planteamiento de hipótesis de causalidad entre una o más dimensiones y las restantes. Ello conlleva el carácter asimétrico del diseño, dado que, de acuerdo con la hipótesis, mientras alguna de las dimensiones tendrá el carácter de agente causal, otras asumirán el de consecuencia. Por tanto, se establece una direccionalidad determinada entre dichas dimensiones. El desarrollo analítico adecuado es el análisis logit, que es una variante del análisis log-lineal.

\subsection{Cuadrante IV: Diseños diacrónico/sincrónicos, o lag-log}

Los diseños mixtos, por su ubicación en el IV cuadrante, se caracterizan por el seguimiento de una pluralidad de unidades, que puede tratarse de diversas dimensiones en un bebé, o en varios, o la relación entre diversos bebés respecto a una acción común del programa.

Indudablemente, se trata de un problema complejo a desglosar:

a) Por una parte, desde un acercamiento al cuadrante I, cabría realizar el estudio de la secuencialidad en paralelo a cada uno de los integrantes de la pluralidad de unidades (bebé o adulto), y que, como caso particular, podría dar lugar en algunas ocasiones a la designación de un sujeto prototípico que pudiera considerarse representativo de todos los que mantuvieran los atributos o características de membrecía respecto al grupo inicialmente definido.

b) También es posible enfocar el estudio desde un acercamiento al cuadrante III, considerando el cruzamiento mencionado anteriormente no en una única ocasión, sino una sucesión de análisis puntuales cercanos en el tiempo, lo que implicaría un cuasi-seguimiento a lo largo del período de tiempo considerado. Es decir, en un momento inicial, y mediante un analisis log-lineal, se conoce cuál es la relación entre los diversos niveles de respuesta contemplados, ope- 
ración que se repite, periódicamente o no, siempre que se juzgue relevante, y que puede llegar a realizarse incluso en cada una de las sucesivas sesiones de registro. Las enormes posibilidades de este diseño diacrónico/sincrónico permiten que se le pueda considerar como el más completo y óptimo para la evaluación del comportamiento, hasta el punto que todos los demás serían variantes incompletas de este.

Estos diseños, como su nombre indica, combinan las dimensiones diacrónica y sincrónica (de aquí la expresión lag-log, resultante de combinar el término emblemático lag -propio de los diseños diacrónicos- con el término emblemático log propio de los diseños sincrónicos-), y se pueden conceptualizar como el estudio relacional de múltiples unidades (niveles de respuesta y/o individuos) a lo largo de los sucesivos momentos temporales (registrados extensiva o intensivamente) que conforman el seguimiento (Bakeman, Adamson \& Strisik, 1989). Resulta obvio considerar que se pueden presentar diversas modalidades que se ajustan a este planteamiento propio de los diseños mixtos, dando lugar al desarrollo de 24 diseños en este IV Cuadrante.

En definitiva, resaltamos la extraordinaria relevancia de los diseños lag-log, que se manifiestan como los diseños observacionales de mayor potencia y los más completos, gracias precisamente a la complementariedad de criterios en los que se basan. Podríamos afirmar con contundencia que se trata de los diseños más completos, al aunar características propias de los diacrónicos y de los sincrónicos, y que todos los demás diseños observacionales podrían considerarse como variantes incompletas de los diseños lag-log.

\section{Referencias Bibliográficas}

AGUIRRE JAIME, A. (1994). Introducción al tratamiento de series temporales. Aplicación a las Ciencias de la Salud. Madrid: Díaz de Santos.

ANGUERA, M. T. (1979, Abril). Observación de la conducta espacial. VI Congreso Nacional de Psicología. Pamplona.

ANGUERA, M. T. (1985). Metodología de la observación en las Ciencias Humanas ( $3^{\text {a }}$ edic. ampliada). Madrid: Cátedra

ANGUERA, M. T. (1991). Proceso de categorización. En M.T. Anguera (Ed.), Metodología observacional en la investigación psicológica (pp. 115-167). Barcelona: P.P.U., vol. I.

ANGUERA, M. T. (1995). Diseños. En R. Fernández-Ballesteros (Ed.), Evaluación de programas sociales: Una guía práctica en ámbitos sociales, educativos y de salud (pp. 149-172). Madrid: Síntesis.

ANGUERA, M. T., BEHAR, B., BLANCO, A., CARRERAS, V., LOSADA, J. L., QUERA, V. y RIBA, C. (1993). Glosario. En M.T. (Ed.), Metodología observacional en la investigación psicológica (pp. 587-617). Barcelona: P.P.U., vol. II.

ANGUERA, M. T., BLANCO, A., LOSADA, J. L. y SÁNCHEZ-ALGARRA, P. (1999). Análisis de la competencia en la selección de observadores. Metodología de las Ciencias del Comportamiento, 1 (1), 95-114. 
BAKEMAN, R. (1978). Untangling streams of behavior: Sequential analyses of observation data. In G.P. Sackett (Ed.), Observing behavior. Vol. II: Data collection and analysis methods (pp. 63-78). Baltimore: University Park Press.

BAKEMAN, R., ADAMSON, L. A. \& STRISIK, P. (1989). Lags and logs: Statistical approaches to interaction. In M.H. Bornstein \& J. Bruner (Eds.), Interaction in human development (pp. 241-260). Hillsdale, N.J.: Lawrence Erlbaum Associates.

BAKEMAN, R. \& DABBS, J. M. (1976). Social interaction observed: Some approaches to the analysis of behavior streams. Personality and Social Psychology Bulletin, $2,335-345$.

BAKEMAN, R. y GOTTMAN, J. M. (1989). Observación de la interacción: Introducción al análisis secuencial. Madrid: Morata (ed. orig., 1986).

BAKEMAN, R. \& GOTTMAN, J. M. (1997). Observing behavior. An introduction to sequential analysis, 2nd ed. Cambridge: Cambridge University Press.

BAKEMAN, R. \& QUERA, V. (1992) SDIS: A sequential data interchange standard. Behavior Research Methods, Instruments \& Computers, 24 (4), 554-559.

BAKEMAN, R. y QUERA, V. (1996). Análisis de la interacción. Análisis secuencial con SDIS y GSEQ. Madrid: Ra-Ma.

BAKEMAN, R., QUERA, V., MCARTHUR, D. \& ROBINSON, B. (1997). Detecting sequential patterns and determining their reliability with fallible observers. Psychological Methods, 2, 357-370.

BEHAR, J. y RIBA, C. (1993) Sesgos en metodología observacional. En M.T. Anguera (Ed.) Metodología observacional en la investigación psicológica (pp. 9-148). Barcelona: P.P.U., Vol. II.

BISHOP, Y. M., FIENBERG, S. E. \& HOLLAND, P. W. (1975). Discrete multivariate analysis. Cambridge, Mass.: The M.I.T. Press.

BLANCO, A. (1989). Fiabilidad y generalización de la observación conductual. Anuario de Psicología, 43 (4), 5-32.

BLANCO, A. (1993). Fiabilidad, validez, precisión y generalización de los diseños observacionales. En M.T. Anguera (Ed.), Metodología observacional en la investigación psicológica (pp. 149-274). Barcelona: P.P.U.

BLANCO, A. (1997). Metodologies qualitatives en la investigació psicològica. Barcelona: Edicions de la Universitat Oberta de Catalunya.

BLANCO, A. y ANGUERA, M. T. (1991). Sistemas de codificación. En M.T. Anguera (Ed.), Metodología observacional en la investigación psicológica (pp. 193-239). Barcelona: P.P.U., vol. I.

CAMBRODÍ, A. y SASTRE, S. (1993). Escales d'observació sistemática (0;0 - 3;0 anys). Barcelona: P.P.U.

CONDON, W. J. \& OGSTON, W.D. (1967) A segmentation of behabior. Journal of Psychiatric Research, 5, 221-235.

ESCUDERO, V. (1995). Análisis secuencial de retardo. Manuscrito no publicado. Coruña: Universidad de La Coruña. 
FIENBERG, S. E. (1977). The analysis of cross-classified categorical data. Cambridge, Mass.: The M.I.T. Press.

GOODMAN, L. A. (1970). The multivariate analysis of qualitative data: Interactions among multiple classifications. Journal of the American Statistical Association, 65, 226-256.

GOODMAN, L. A. (1971). The analysis of multidimensional contingency tables: Stepwise procedures and direct estimation methods for building models for multiple classifications. Technometrics, 13, 33-61.

KENNEDY, J. J. (1983). Analyzing qualitative data. Introductory log-linear analysis for behavioral research. New York: Praeger.

KRATOCHWILL, T. R. (Ed.) (1978). Single subject research. Strategies for evaluating change. New York: Academic Press.

KRITZER, H. M. (1986). Using categorical regression to analyze multivariate contingency tables. In W.D. Berry \& M.S. Lewis-Bech (Eds.), New tools for social scientists. Advances and applications in research methods (pp. 157-201). Beverly Hills: Sage.

LAREO, S. (1984). Enfermas mentales crónicas: Un estudio ecológico y conductual en una alternativa de asistencia comunitaria. Tesis de Licenciatura no publicada. Barcelona: Universidad de Barcelona.

LOSADA, J. L. (1993) Instrumentos de la observación. En M.T. Anguera (Ed.) Metodología observacional en la investigación psicológica (pp. 263-340). Barcelona: P.P.U., Vol. II.

LOSADA, J. L. (1995). Análisis secuencial. Manuscrito no publicado. Barcelona: Universidad de Barcelona.

LOSADA, J. L. (1999). Metodología observacional. A Coruña: Penta.

MAGNUSSON, M. S. (1993). THÈME user's manual: With notes on theory, model and pattern detection method. Reykjavik: University of Iceland.

MAGNUSSON, M. S. (1996). Hidden real-time patterns in intra- and inter-individual behavior. European Journal of Psychological Assessment, 12 (2), 112-123.

MAGNUSSON, M. S. (2000). Discovering Hidden Time Patterns in Behavior: TPatterns and their detection. Behavior Research Methods, Instruments \& Computers, 32 (1), 93-110.

MAGNUSSON, M. S. \& BEAUDICHON, J. (1997). Détection de 'marqueurs' dans la comunication référentielle entre enfants. En J. Bernicot, J. Caron-Parque et $A$. Trognon (Eds.), Conversation, Interaction et Fonctionement Cognitif (pp. 315 335). Nancy: Presse Universitaire de Nancy.

MONTAGNER, H., MAGNUSSON, M. S., CASAGRANDE, C., RESTOIN, A., BEL, J.P., NHUYEN, M., H. PAUL, RUIZ, V., DELCOURT, S., GAUFEIER, G. \& EPOULET, M. (1990). Une nouvelle méthode pour l'étude des organisateurs de comportement et systèmes d'interaction du jeune enfant. Psychiatrie de l'Enfant, $33,391-456$. 
MORALES, M. (1996). Análisis secuencial. Manuscrito no publicado. Sevilla: Universidad de Sevilla.

MUCCHIELLI, R. (1974). L'observation psychologique et psychosociologique. Paris: ESF.

MURILLO, C. (1994). Métodos estadísticos de series temporales. Aplicaciones sanitarias. Barcelona: SG.

PASTOR, E. y SASTRE, S. (1999). Patrones de interacción adulto-niño en la construcción del significado: Aplicación del programa "Thème". En M.T. Anguera (Coord.), Observación de conducta interactiva en contextos naturales: Aplicaciones (pp. 125-150). Barcelona: EUB.

QUERA, V. (1993). Análisis secuencial. En M.T. Anguera (Ed.), Metodología observacional en la investigación psicológica (pp. 341-586). Barcelona: P.P.U.

SACKETT, G. P. (1980). Lag sequential analysis as a data reduction technique in social interaction research. In D. B. Sawin, R. C. Hawkins, L. O. Walker \& J. H. Penticuff (Eds.), Exceptional infant. Psychosocial risks in infant-environment transactions (pp. 300-340). New York: Brunner/Mazel.

SACKETT, G. P. (1987). Analysis of sequential social interaction data: Some issues, recent developments, and a causal inference model. In J.D. Osofsky (Ed.), Handbook of infant development (pp. 855-878). New York: Wiley.

SASTRE, S. y PASTOR, E. (1999). Observación del desarrollo cognitivo temprano: Propuesta de análisis cualitativo. En M.T. Anguera (Coord.), Observación en la escuela: Aplicaciones (pp. 249-279). Barcelona: E.U.B.

WEICK, K. E. (1968). Systematic observational methods. In G. Lindzey \& E. Aronson (Eds.), Handbook of Social Psychology (pp. 357-451). Reading, Mass.: AddisonWesley, vol. II. 